\title{
La nueva asignatura de educación ciudadana en Chile: creencias de profesores y profesoras
}

\author{
The new subject of citizenship education in Chile: teachers' belief \\ Paula Soto Lillo ${ }^{a}$ \& Cinthia Peña Hurtado ${ }^{b}$ \\ aPontificia Universidad Católica de Valparaíso, Chile. \ paula.soto.l@pucv.cl \\ [orcid.org/0000-0002-0426-4009] \\ bPontificia Universidad Católica de Valparaíso, Chile. cinthia.pena@pucv.cl \\ [orcid.org/0000-0002-6390-482X]
}

\section{RESUMEN}

Los y las profesores de Historia están encargados de impartir la nueva asignatura Educación Ciudadana, sin embargo, la investigación ha establecido que la Formación Inicial Docente en Chile es insuficiente en temáticas de ciudadanía. Considerando esto, se realizó una encuesta de opinión a un total de 50 docentes sobre esta nueva asignatura, su capacidad profesional para enseñarla y las principales facilidades y dificultades que encuentran para dictarla. Los resultados arrojan que la mayoría de quienes participaron tienen una visión positiva sobre la asignatura, aunque son críticos del programa presentado por el Ministerio de Educación. Gran parte de los y las docentes no tienen certeza de tener suficientes herramientas profesionales. No obstante, la formación continua y la experiencia se constituyen en un aspecto clave que permite suplir las deficiencias de formación inicial. Finalmente, la mayoría se plantea frente a la implementación de este nuevo currículo en una postura de adaptación creativa.

PALABRAS CLAVE: Educación Ciudadana, nuevo currículo, creencias docentes.

\section{ABSTRACT}

History teachers are in charge of teaching the new subject of Citizenship Education, however various studies have established that Initial Teacher's Education in Chile is insufficient in citizenship issues. An opinion survey was carried out among a total of 50 history teachers regarding this new subject, their professional ability to delivering lessons, and the main facilities and difficulties they encounter in teaching it. The results show that 
most of those who participated have a favourable view of the subject, although they are critical of the program presented by the Ministry of Education. Most of the teachers are not sure they have enough professional tools. Still, continuous training and experience are crucial aspects that allow them to overcome the deficiencies of initial training. Finally, they face the implementation of this new curriculum in a position of creative adaptation.

KEY WORDS: Citizenship Education, New curriculum, teacher beliefs

\section{INTRODUCCIÓN}

La complejidad de los desafíos que enfrenta nuestra democracia, como el progresivo distanciamiento entre la ciudadanía y la política, la desafección de instituciones como partidos políticos o la escasa participación juvenil en instancias de elección de cargos de votación popular, han influido en que la educación y formación ciudadana se instale en la agenda pública. No solo a nivel de demandas de la ciudadanía, sino, además, desde una iniciativa estatal que busca por medio de reformas curriculares, leyes y programas, fortalecer una educación cívica y ciudadana (Kerr, 2015). Desde el ámbito de la política educativa nacional, aun cuando el problema es de larga data -ya que desde inicios de siglo existen experiencias de inclusión de contenidos cívicos y ciudadanos en el currículo escolar-, en la implementación asignaturas atingentes "se han sucedido diferentes enfoques respecto de la manera en cómo esta es aplicada en los establecimientos, tanto en sus contenidos como en sus metodologías" (Agencia de Calidad de la Educación, 2016, p.7). Siendo los últimos años, aquellos donde la Educación Ciudadana ha cobrado mayor interés, conforme la importancia que esta posee en la formación de futuros ciudadanos, la constituye como un factor clave en la comprensión de una democracia más profunda y compleja desde temprana edad.

Si bien, la dictación de esta asignatura viene mandatada por la Ley 20.911 y su programa viene establecido desde el Ministerio de Educación (MINEDUC), sabemos que a los sistemas educativos le corresponde una importante responsabilidad en la formación de los ciudadanos, puesto en el espectro privado de la vida familiar u otras asociaciones, no se aprende automáticamente a serlo. Así entonces, profesores y profesoras de Historia y Geografía, asumen la responsabilidad de desarrollar la asignatura de Educación Ciudadana en establecimientos escolares y con ello, promover el desarrollo de conocimientos y competencias cívicas que posibiliten el ejercicio y comprensión de una ciudadanía democrática, entendiendo que es la escuela el lugar "donde se rompe con la incondicionalidad del hogar y se accede a un nosotros, amplio o restringido, que es base de la vida cívica" (Cox \& Castillo, 2015, p. 7). Por lo mismo es fundamental preguntar a los y las docentes como perciben este nuevo programa de Educación Ciudadana.

La Educación Ciudadana está a cargo de los y las profesores de Historia, sin embargo, hay un aspecto esencial que es necesario considerar. La Formación Inicial de profesores y profesoras de Historia en Chile en materias de ciudadanía resulta insuficiente, debido a la escasez de asignaturas orientadas a este objetivo, aspecto que ha sido estudiado por distintas investigaciones (Mardones et al. 2014; Agencia de la Calidad de la Educación, 2016; Altamirano, 2018). 
Considerando estos resultados, planteamos que es posible establecer que las y los profesores de Historia no están suficientemente preparados para hacerse cargo de estas materias, por lo que la implementación del curso requiere que los y las docentes busquen de forma autónoma como hacerse cargo de la asignatura. A pesar de la dificultad que implica la falta de preparación, los y las docentes están altamente dispuestos a capacitarse (Mardones et al. 2014) lo que presenta un aspecto positivo frente a la implementación de la asignatura.

En función de lo anterior, en este trabajo se busca analizar las percepciones que tienen docentes en ejercicio sobre la asignatura de Educación Ciudadana y las condiciones profesionales que poseen y en que se encuentran para dictarla. A partir de ello, las preguntas que guían la presente investigación son: ¿Qué opinión tienen profesores y profesoras de Historia y Geografía sobre el programa de estudio de Educación Ciudadana? ¿Qué tan capacitados se sienten los profesores y profesoras de Historia y Geografía para impartir la asignatura de Educación Ciudadana considerando su formación inicial docente? ¿Cuáles son las principales dificultades y facilidades que los profesores y profesoras de Historia y Geografía tienen para la implementación de la asignatura de Educación Ciudadana, considerando su formación inicial docente?

\section{MARCO REFERENCIAL}

\section{La Educación Ciudadana en el Currículo}

Con la aprobación de la Ley Orgánica Constitucional de Enseñanza (LOCE) queda establecido un Marco Curricular obligatorio para establecimientos escolares, con Objetivos Fundamentales y Contenidos Mínimos Obligatorios, de la Educación Básica (1996) y la Educación Media (1998). Estos plantean para la ‘Educación Ciudadana’ nuevas demandas que "requieren pasar desde la tradicional educación cívica planteada en una asignatura en Educación Media, referida a conocimientos sobre la estructura y funcionamiento del sistema de gobierno, a una formación ciudadana, más inclusiva y más profunda" (Cox, 2011. p. 6). Esta nueva perspectiva determina un cambio posicional en el currículo, con la inclusión de nuevas asignaturas como 'Comprensión del Medio Natural, Social y Cultural'; 'Estudio y Comprensión de la Sociedad' y ‘Orientación', junto a la eliminación de ‘Educación Cívica y Economía’ para implementar sus contenidos en las asignaturas de 'Historia' y 'Filosofía y Psicología'. Estos determinaron que la enseñanza y el aprendizaje de los contenidos de Educación y Formación Ciudadana se distribuyeran de manera transversal en diversas asignaturas y niveles, junto a Objetivos de Aprendizaje Transversal afines a todas las áreas.

Respecto a la concepción de ciudadanía concebida en este nuevo currículo, observamos la incorporación de una formación ciudadana que transita de la educación cívica planteada como única asignatura del currículo, a una comprensión y formación ciudadana acorde al contexto de democratización y globalización actual, más profunda en términos de habilidades, valores, actitudes y conocimientos para la vida en democracia, es decir, una articulación entre "conocimiento sobre instituciones y procedimientos de la ciudadanía democrática, por un lado, con las habilidades requeridas por la misma en la situación presente, por otra" (Bascopé, Cox \& Lira, 2015, citado en Cox \& Castillo, 2015, p. 256). 
Kerr (1999, 2002) plantea que existen dos grandes enfoques asociados a la formación de la ciudadanía; una minimalista y una maximalista; por la cual entendemos dos comprensiones que nos permiten definir qué tipo de relación existe entre la Educación y la ciudadanía. En la primera, predomina una dimensión cívica tradicional, donde la Educación está centrada en el conocimiento de contenidos factuales, tales como la institucionalidad política o los orígenes de la democracia. En oposición, una comprensión maximalista pone énfasis en una ciudadanía amplia y activa, la cual trasciende al aprendizaje de contenidos conceptuales para posibilitar experiencias de aprendizaje de habilidades y actitudes, ya sea en diversas asignaturas del currículo, o experiencias de aprendizaje democrático donde se insertan estudiantes, por ejemplo a consejos de curso o centros de alumnos.

Tras diez años de implementación, se realizarán dos modificaciones más al currículo de ciudadanía. A saber, una instaurada en el año 2009 (siendo preconcebida desde 2006, con atención al Informe de la Comisión Ciudadana elaborado en 2004). Y otra, en 2013. El primero de los cambios, refiere al ajuste de los marcos curriculares existentes (Enseñanza Básica y Enseñanza Media), “que significó el fortalecimiento de la presencia de la formación ciudadana, por densificación y especificación de objetivos y contenidos" (Cox \& García, 2015, citado en Cox \& Castillo, 2015, p. 290). En 2013, en cambio, las modificaciones se traducen en la definición de Bases Curriculares y Objetivos de Aprendizaje que reemplazan a los OF y CMO, tras la reformulación a su vez, de la LOCE por la Ley General de Educación de 2009, los cuales, constituyen modificaciones a la secuencia y articulación de contenidos desde $1^{\circ}$ año de Enseñanza Básica a $2^{\circ}$ año de Enseñanza Media. Así es como se implementan mayores oportunidades de aprendizaje de ciudadanía, conforme se incorpora el eje de formación ciudadana dentro de la asignatura de Historia, Geografía y Ciencias Sociales desde $1^{0}$ año de Enseñanza Básica hasta $2^{\circ}$ año de Enseñanza Media y nuevos Objetivos de Aprendizaje Transversales ('dimensión sociocultural y ciudadana y dimensión moral', que vienen a suplantar los OFT de 'formación ética y persona y entorno').

La segunda modificación en 2015, tras las recomendaciones elaboradas por el 'Consejo Asesor Presidencial contra Conflictos de Interés, el Tráfico de Influencias y la Corrupción’, apela nuevamente a la importancia de la formación ciudadana en etapas escolares, debido a lo cual se promulga la Ley 20.911 del año 2016, que da origen al Plan de Formación Ciudadana para todos los establecimientos que reciben financiamiento público en Chile. La cual señala para los niveles de educación parvularia, básica y media, la inclusión de experiencias de aprendizaje y formación ciudadana que brinden

la preparación necesaria para asumir una vida responsable en una sociedad libre y de orientación hacia el mejoramiento integral de la persona humana, como fundamento del sistema democrático, la justicia social y el progreso. (...) propender a la formación de ciudadanos, con valores y conocimientos para fomentar el desarrollo del país, con una visión del mundo centrada en el ser humano, como parte de un entorno natural y social (p. 1). 
Mandato que se suma, además, a la creación de una nueva asignatura de Educación Ciudadana, para los niveles finales del ciclo escolar $-3^{\circ}$ y $4^{\circ}$ año medio- de Formación General Artística, Técnico Profesional y Humanístico Científica a partir de 2020, a cargo de profesores y profesoras de Historia y Geografía. El objetivo de esta nueva asignatura es

(...) desarrollar un conjunto de conocimientos, habilidades y actitudes para que los estudiantes conozcan el sistema democrático y se desenvuelvan en él, participando en forma activa y corresponsable en la construcción de una sociedad orientada hacia el fortalecimiento del bien común, la justicia social y el desarrollo sustentable" (Ministerio de Educación, 2019, p. 38).

En suma, el currículo de Educación y Formación Ciudadana pretende una comprensión que busca dar respuesta a la demanda de formación de ciudadanos activos, en instituciones escolares que privilegien la vivencia de la democracia, la identificación con la comunidad y el aprendizaje de habilidades y actitudes positivas en torno a estas, "ciudadanos críticos, creativos y reflexivos, activamente participativos, solidarios y responsables, con conciencia de sus deberes y derechos, y respeto por la diversidad de ideas, formas de vida e intereses" (Ministerio de Educación, 2020. p. 7).

\section{Creencias docentes}

Las creencias docentes constituyen un aspecto ampliamente documentado por la literatura (Pajares, 1992; Calderhead, 1996; Roehrig, Kruse \& Kern, 2007; Rodríguez \& Solis, 2017) y a partir de ello se concluye que la posición que finalmente asuman profesores y profesoras frente a un nuevo currículum estará directamente influida por sus creencias personales (Wallace \& Priestley, 2011). Esto tiene consecuencias a la hora de implementar los programas de estudio, pues frente a una misma política pública, podemos encontrar variedad de interpretaciones de este por parte de los y las docentes.

Las creencias docentes filtran, enmarcan y guían sus percepciones y la implementación de los currículos (Fives \& Buehls, 2016). Un ejemplo concreto de esta influencia, lo encontramos en la investigación de Tham (2014) quien, al analizar las perspectivas de profesores de Historia chilenos sobre la Educación Ciudadana, levantó una diversidad de categorías que dan cuenta de una variedad de concepciones sobre el tema.

Las concepciones sobre formación ciudadana entran en la categoría de lo que Shulman (1986) denomina conocimiento pedagógico del contenido, constituyendo así, una de las dimensiones de las creencias docentes.

Los resultados de Tham (2014) son relevantes, pues dan cuenta de las creencias conceptuales sobre Formación Ciudadana de quienes están encargados de implementar el currículum escolar. Dada la diversidad de creencias al respecto, la implementación de esta asignatura tendrá distintos énfasis, pudiendo variar respecto al sentido original que propone el currículo escolar oficial. 


\section{Implementación de nuevos currículos}

La implementación de nuevos programas y currículos escolares ha sido tema de estudio a lo largo de los años al evaluar su efectividad. Como ya hemos señalado, las creencias juegan un papel fundamental. Según Edwards (2010) los y las docentes antes de implementar un nuevo currículum, lo traducen, o según Wallace y Priestley (2011), lo transforman a partir de sus propias creencias. A partir de ello, la literatura ha establecido que los profesores son claves para el éxito de las reformas curriculares (Bantwini, 2010), u obstáculos para el cambio (Handal \& Herrington, 2003), no obstante, existen otros factores que también inciden en esta comprensión (Fullan, 2001).

Ávalos (2006) plantea que la implementación de nuevos currículos da origen a distintas tensiones. Una primera gran tensión corresponde a la expectativa de que los y las docentes sean agentes de la implementación de un currículum determinado por el sistema y del cual, no necesariamente fueron constructores. En ese sentido, Fullan (2002) sostiene que cambios propuestos a los y las docentes desde fuera, tienen dificultades para llegar a la sala de clases. Esta primera tensión se ve claramente ejemplificada por Cox (2011) quien señala que, para el caso del ajuste curricular de 2009, si bien hubo instancias de participación docente, este finalmente no fue consultado generando críticas por parte del profesorado.

Ante la falta de participación en la elaboración del currículum, algunos docentes pueden plantearse en dos extremos, pasivos o resistentes frente a la implementación, o generar una respuesta de adaptación creativa (Ávalos, 2006). En concordancia con lo señalado por la autora, Spillane y colaboradores (2002) explican que puede darse el caso que los y las docentes no sean capaces de leer correctamente la política pública, ignorarla de forma intencional o, seleccionar aquellas que sean coincidentes con sus propios intereses, lo que lleva a que no se realice una implementación de acuerdo con el espíritu de los creadores del programa, sino de acuerdo con las creencias de cada profesor.

Para Ávalos (2006), una segunda tensión se genera producto de los contextos en los que los y las docentes se desempeñan. Laura (2005) plantea que el currículo que profesores y profesoras implementan, se ve limitado por sus posibilidades, recursos académicos y por el tiempo del que disponen, los que se convierten en aspectos de resistencia (De Alba, 1991). En ese sentido, Fullan (2001) sostiene que las reformas educativas requieren enseñanza de calidad y materiales de enseñanza, de lo contrario no será posible un cambio sustancial. Por lo mismo, el conocimiento y la preparación docente son fundamentales a la hora de llevar a la sala de clases un nuevo currículo escolar. En torno a esta idea, Cox (2011), señala que justamente el problema central de la implementación del currículum escolar chileno del 2009 se relacionaba con las capacidades docentes, pues estas estaban más relacionadas con las necesidades de ampliar la cobertura del sistema educacional chileno, que con la calidad de éste.

\section{METODOLOGÍA}

En cuanto a la metodología, el presente estudio es de carácter mixto y su diseño corresponde al de encuesta de opinión de carácter exploratorio (Hernández, Fernández \& 
Baptista, 2014), por cuanto este procedimiento "permite obtener y elaborar datos de modo rápido y eficaz" (Casas, Repullo \& Campos, 2003. p. 527). La encuesta considera un ítem de caracterización sobre los años de ejercicio profesional y luego un total de 8 preguntas abiertas y cerradas. La elaboración de este instrumento consideró la construcción de un instrumento de validación (Thomas, 2017) el cual fue entregado a un grupo de 3 expertos integrado por 1 docente de Historia que se desempeña en el mundo escolar, un académico universitario que trabaja temas de ciudadanía y una académica del área de Metodología de la Investigación. A partir de esta validación, se realizaron ajustes a las preguntas que dieron origen al instrumento final que se compartió con los y las docentes participantes del estudio.

La población del estudio está constituida por profesores y profesoras de la especialidad de Historia y Geografía. La muestra se realizó mediante autoselección (Rocco \& Oliari, 2007), alcanzando un total de cincuenta docentes, que participaron de forma voluntaria en el estudio. De acuerdo con el reporte del Centro de Estudios Mineduc (2019) en Chile hay 6.695 profesores y profesoras de Historia ejerciendo en Chile, por tanto, esta muestra representa el 0,75\% del universo de docentes de la disciplina. Debido a su tamaño, esta muestra no busca ser representativa del universo de profesores.

La encuesta fue aplicada durante los meses de mayo y junio de 2020, a través de un formulario de Google, el cual recogió las respuestas de forma anónima.

Todas las preguntas del cuestionario se encuentran íntegramente explicadas en el apartado de resultados. Para el análisis de las respuestas a las preguntas abiertas, se realizó una categorización o transformación de la información textual en dato (Sánchez, Delgado \& Santos, 2013) considerando las preguntas de investigación. Posterior a ello, se identificaron las palabras clave para cada categoría y se procedió a codificar la información. Con dicha codificación se pudieron realizar nubes de palabras en función de la frecuencia, las que son presentadas en el apartado de resultados.

En tanto las preguntas de respuesta cerrada fueron graficadas para representarlas visualmente.

\section{RESULTADOS}

Para caracterizar a los y las participantes, se les solicitó indicar cuántos años de ejercicio profesional poseían. Respondieron un total de 50 docentes, cuya experiencia laboral va desde el primer año, hasta los 33 de ejercicio profesional (Fig. 1). Del total de estos participantes, el 48\% está dictando la asignatura, mientras que el 52\% restante, declara no dictarla actualmente.

Para indagar sobre las opiniones que docentes tienen sobre la asignatura de Educación Ciudadana, se realizó la pregunta 2 de tipo abierta, 'Independientemente si lo dicta o no ¿cuál es su opinión sobre el programa ministerial para el curso de Educación Ciudadana?' posibilitando exponer todo aquello que cada participante estimara conveniente. Las respuestas obtenidas resultan variadas desde distintos puntos de vista.

En cuanto a extensión, hay algunas muy acotadas que consideran solo una palabra, hasta otras en que los y las docentes se explayan dando cuenta de un análisis profundo del programa de la asignatura. En cuanto a contenido, se establecieron cinco categorías cuyos 


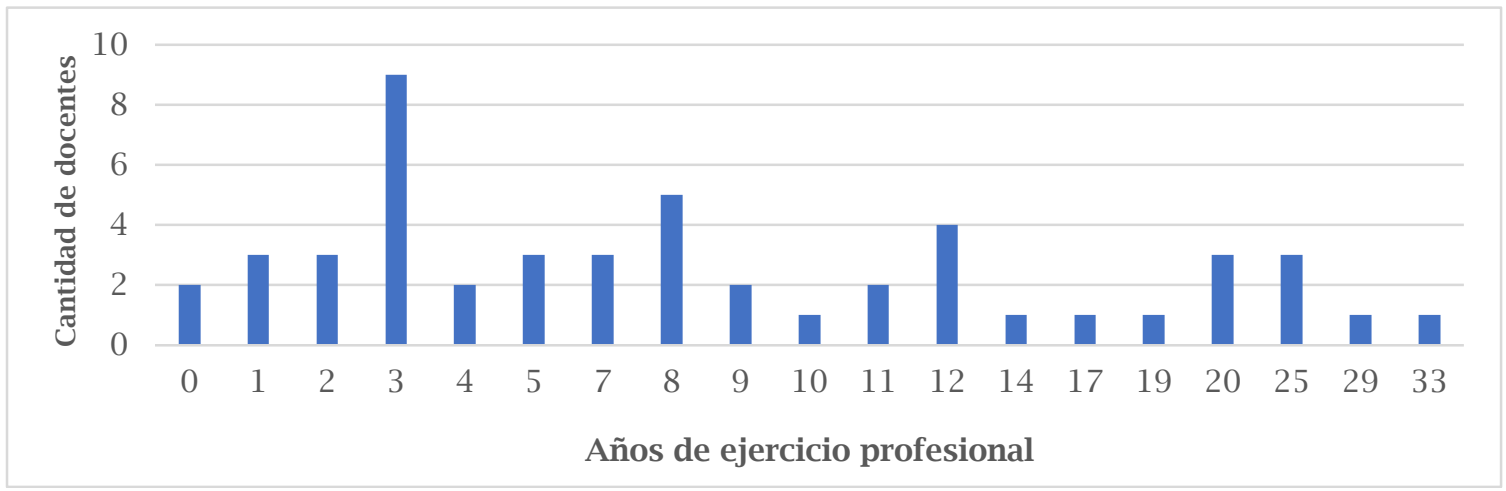

Fig. 1. Años de ejercicio docente de los participantes $(\mathrm{n}=50)$.

Fuente: Elaboración propia.

resultados se agrupan en visiones positivas (46\%), visiones negativas (18\%), visiones positivas con aspectos negativos (24\%), visiones negativas con aspectos positivos (2\%) y visiones neutrales o que no expresan una valoración (10\%), las que se representan en la Fig. 2.
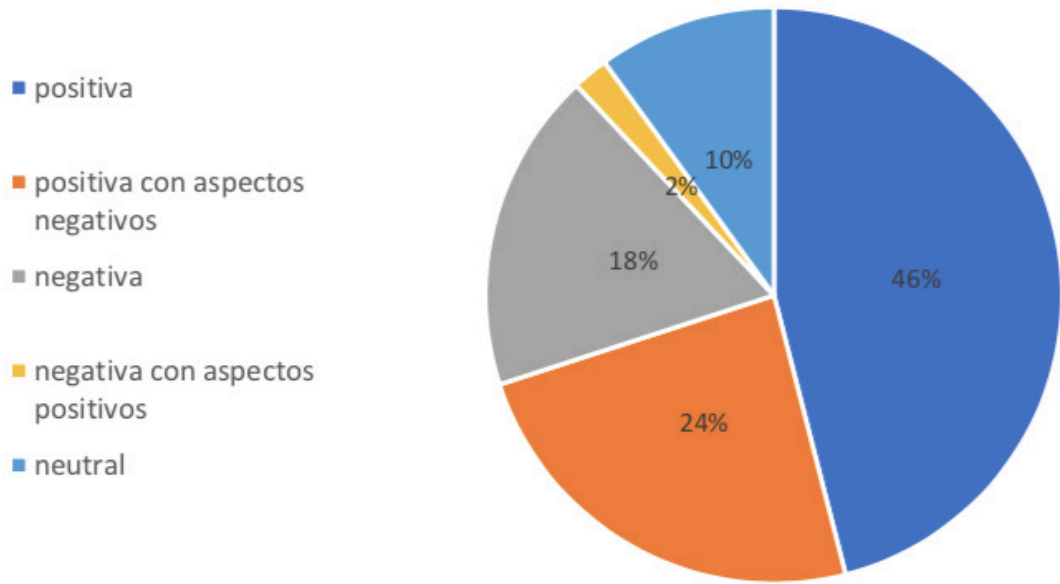

Fig. 2. Opiniones docentes sobre el programa de Educación Ciudadana $(n=50)$. Fuente: Elaboración propia.

En la pregunta 3, se consultó a los y las docentes, 'Independientemente de si lo dicta actualmente o no $i$ Tiene suficientes herramientas profesionales para dictar la asignatura de Educación Ciudadana?'. Esta pregunta cerrada ofrecía tres alternativas de respuesta, la que fue respondida por los 50 participantes, en las que 23 docentes (46\%) señala no tener seguridad, luego 22 (44\%) afirman tener herramientas profesionales y un número minoritario de 5 docentes (10\%) declara que no (Fig. 3).

En la pregunta 4 se le pidió a los y las participantes que justificaran su respuesta anterior a través de una respuesta abierta. Las justificaciones se agruparon en categorías emergentes y para representarlas gráficamente se utilizaron nubes de palabras, donde el tamaño de cada concepto se condice con la cantidad de menciones. 


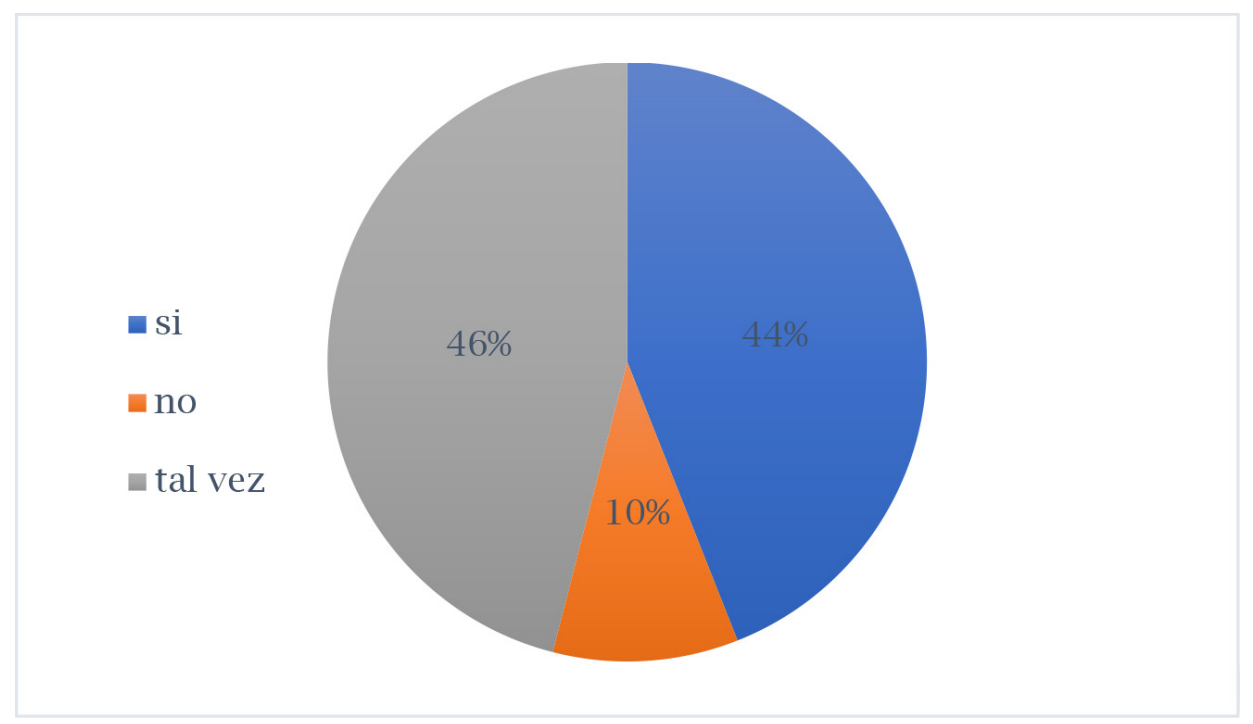

Fig. 3. ¿Tiene suficientes herramientas profesionales para dictar la asignatura de Educación Ciudadana? ( $\mathrm{n}=50)$. Fuente: Elaboración propia.

Entre los 22 docentes que respondieron si, las causas categorizadas que emergen se representan en la Fig. 4. De dichas respuestas hay 9 menciones a tiene suficientes herramientas profesionales gracias a la formación continua, seguida de la experiencia docente con 6 menciones. En tercer lugar, con 5 menciones cada una aparecen la formación inicial docente que según reportan fue apropiada, junto a la similitud de enseñar Historia y Educación Ciudadana como un aspecto que les dio suficientes herramientas. Finalmente, las justificaciones con menos menciones, 3 cada una, dicen relación con poseer suficientes conocimientos y la disponibilidad de recursos disponibles para la enseñanza.

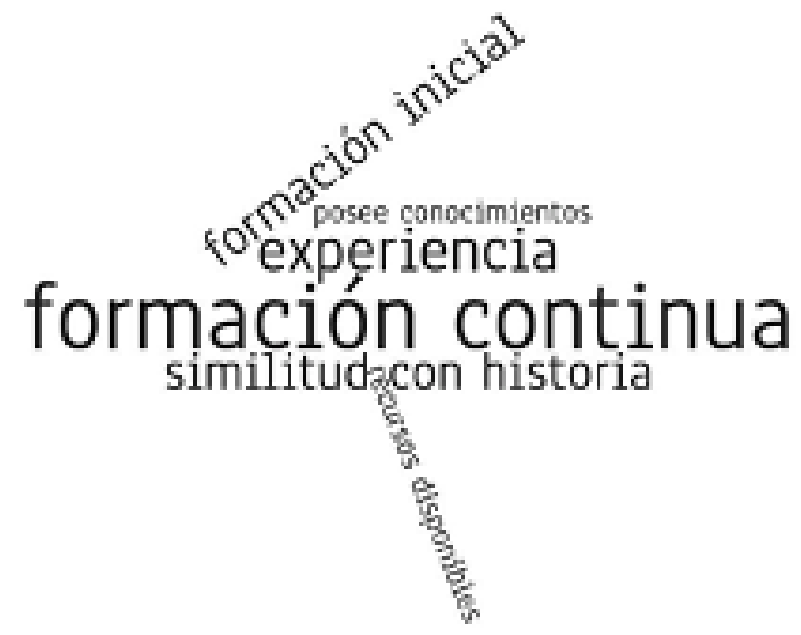

Fig. 4. Justificaciones de la respuesta posee herramientas suficientes $(\mathrm{n}=22)$. Fuente: Elaboración propia. 
Entre quienes respondieron tal vez, las justificaciones son, en primer lugar, porque dicen contar con los conocimientos para enseñar la asignatura con 6 menciones. En segundo lugar, aparece la insuficiencia de la Formación Inicial Docente orientada a la enseñanza de la formación ciudadana con 4 menciones. En tercer lugar, con 3 menciones cada una, encontramos la experiencia, la falta de conocimientos y lo tardío del programa del curso. Con menciones menores aparece la formación continua orientada a la disciplina y la similitud con la enseñanza de la Historia que tienen 2 menciones cada una. (Fig. 5).

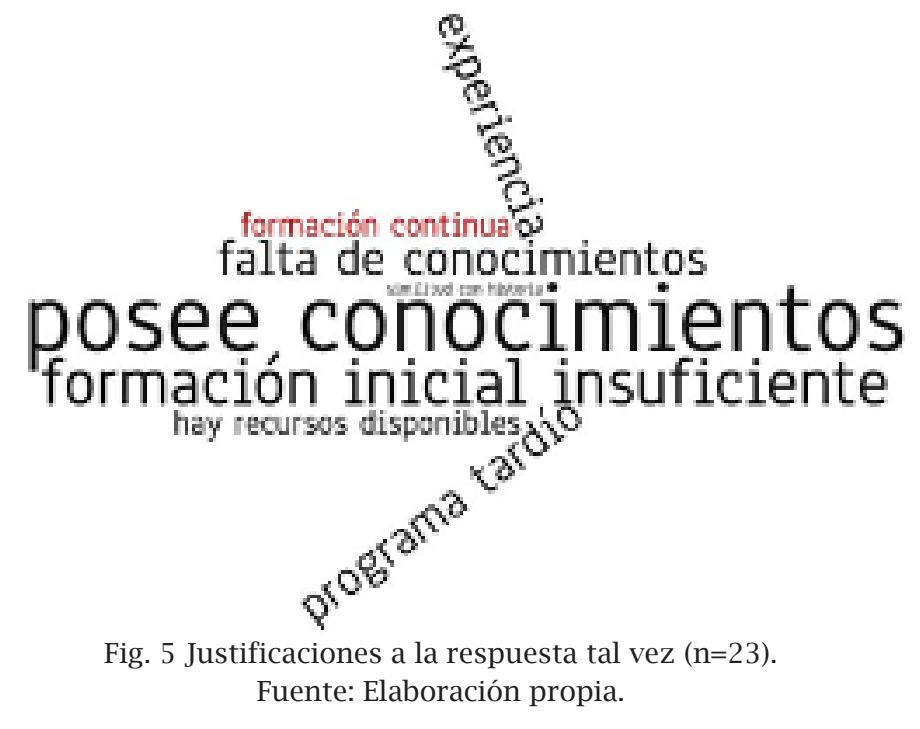

Finalmente, las justificaciones de quienes declaran no tener suficientes herramientas profesionales se dividen por igual entre la deficiente formación inicial docente y la falta de herramientas para enseñar la asignatura con 2 menciones cada una.

La pregunta número 5 '¿Cree usted que dispone de suficientes recursos didácticos para enseñar Educación Ciudadana?’ arrojó resultados divididos por igual entre quienes creen que sí y quienes creen que no, junto a un minoritario 20\% que no cree disponer de suficientes recursos didácticos, tal como se muestra en el Fig. 6.

En torno a la pregunta número 6 “¿podría indicar qué recursos serían pertinentes o necesarios para apoyar la realización del curso?’ a partir de la codificación, la mención más recurrente corresponde a 'fuentes diversas', conforme en 28 oportunidades se les identifica como recursos pertinentes para trabajar en la asignatura de Educación Ciudadana. En este sentido, se enuncia cualquier tipo de recurso -escrito o audiovisualque pueda ser utilizado, sin relación con la promoción de aprendizajes, como es el caso de la distinción posterior 'recursos para a habilidades y actitudes', por la cual existen 19 menciones a diversos tipos de recursos en función de la idoneidad para la promoción de habilidades y actitudes ciudadanas, es decir, facilitadores de aprendizajes en el área. Seguidamente podemos visualizar menciones más acotadas en torno condiciones materiales como 'recursos tecnológicos', cuatro en total. Mientras se presentan solo tres referencias a recursos que promuevan la interdisciplinariedad -desde la utilización de recursos, como desde estrategias didácticas- entre asignaturas. Por último, solo una 


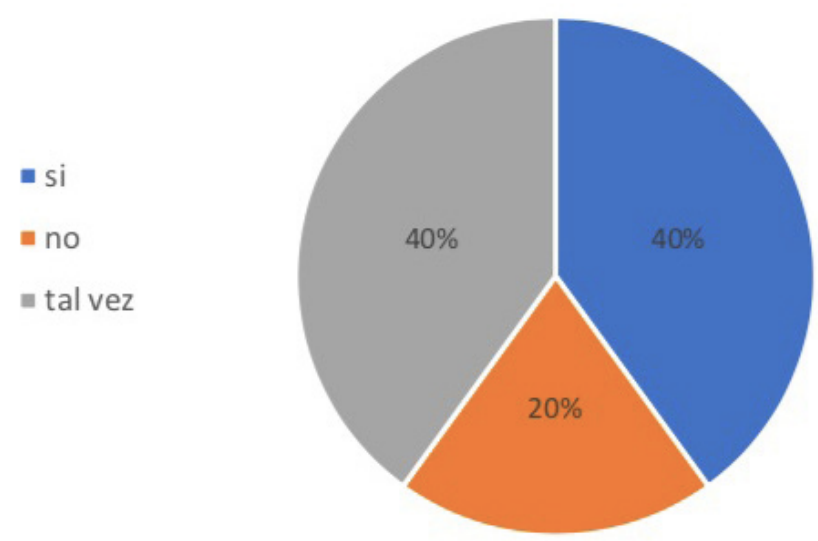

Fig. 6. ¿Cree usted que dispone de suficientes recursos didácticos para enseñar Educación Ciudadana? $(\mathrm{n}=50)$.

Fuente: Elaboración propia.

indicación al ‘tiempo y espacio de planificación' como recurso requerido y pertinente para el desarrollo de la asignatura, así como la inclusión en el Proyecto de Mejoramiento Escolar de recursos para implementarla.

En torno a la pregunta 7 ¿Cuáles son las principales dificultades que tiene para la realización de clases en Educación Ciudadana?' (Fig. 7) destacan en primer lugar, las estrategias de enseñanza y aprendizaje que poseen para esta, conforme 11 referencias les relevan. En segundo lugar, y con igual cantidad de menciones, se indica como obstaculizador la propuesta curricular de la asignatura y las características de estudiantes para su desarrollo, cada una con 10 menciones. Posteriormente se releva las condiciones institucionales y el equipo directivo con ocho referencias y la formación inicial con seis menciones. Surge como dificultad, además, la conectividad con dos menciones.

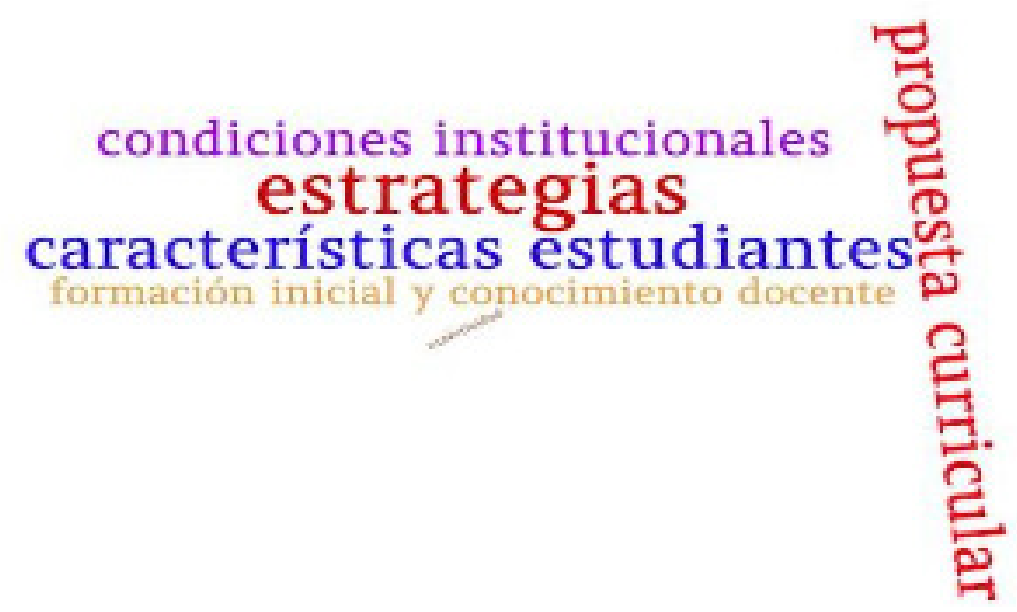

Fig. 7. ¿Cuáles son las principales dificultades que tiene para la realización de clases de Educación Ciudadana? (Independiente del contexto actual de pandemia) $n=50$.

Fuente: Elaboración propia. 
La octava pregunta ‘¿Cuáles son las principales facilidades que tiene para la realización de clases de Educación Ciudadana?’ presenta una amplia tendencia hacia una comprensión del curso como una propuesta programática proclive a la ciudadanía activa con 36 referencias. Identificando además como facilitadores, recursos que se poseen; tanto materiales, como inmateriales; e institucionales, con 12 referencias, así como la formación inicial y los conocimientos profesionales, con 9 distinciones (Fig. 8).

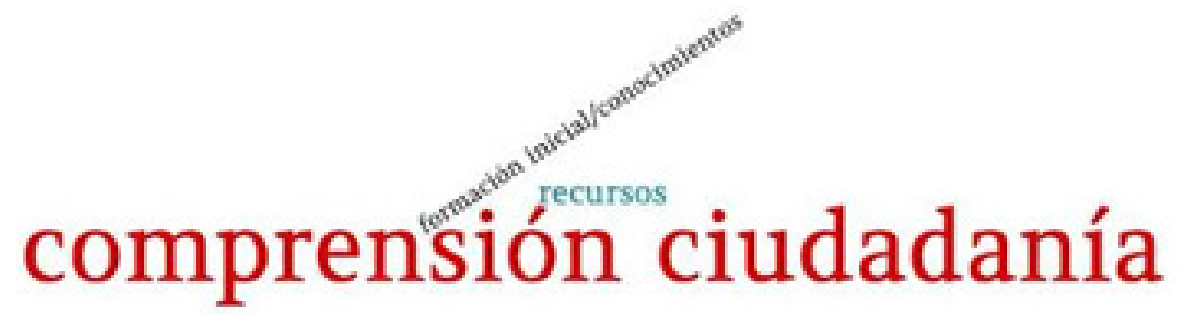

Fig. 8. ¿Cuáles son las principales facilidades que tiene para la realización de clases de Educación Ciudadana? $(n=50)$. Fuente: Elaboración propia.

\section{DISCUSIÓN DE RESULTADOS}

Considerando los resultados presentados en el apartado anterior daremos cuenta de su análisis, en función de las preguntas de investigación que guían el presente este trabajo.

Respecto a la opinión que profesores y profesoras tienen sobre la asignatura de Educación Ciudadana (pregunta 2) las visiones positivas, es decir aquellas que expresan solo aspectos positivos sobre la asignatura, sumadas a las positivas con aspectos negativos (presentan visiones positivas sobre la asignatura, pero agregan elementos que consideran que no están bien), vemos como en conjunto, corresponden a un 70\% de las respuestas. Así, las opiniones negativas corresponden a una minoría, las que, sumadas a la única visión negativa con aspectos positivos, totalizan un 20\%. Finalmente, una minoría de respuestas presentan una visión neutra, es decir, ni positiva ni negativa sobre el programa. Este último tipo de opiniones se fundamenta en el hecho de desconocer el programa y su contenido. La Tabla 1 da cuenta de algunos ejemplos de cada tipo de respuesta.

El hecho de que la gran mayoría de docentes exponga una visión positiva del programa permiten pensar que este grupo de docentes a pesar de no haber participado en la elaboración de este, no se presentan en una posición de resistencia (Ávalos, 2006), sin embargo, es necesario considerar que cada cual lo implementará según sus propias creencias (Edwards, 2010; Wallace \& Priestley, 2011) algo que incluso uno de los docentes expresa abiertamente:

(...) me incomoda mucho que estos nuevos programas no tengan indicadores de evaluación claros para cada objetivo. Esta situación, especialmente cuando se trabaja con una nueva asignatura, dificulta el trabajo docente, vuelve más complejo el panorama al planificar y evaluar, debido a que uno debe inferir en qué debe hacer énfasis y con se evidenciaría" (...) por lo ya mencionado, complejiza la labor docente al tener que definir qué parte del objetivo se espera trabajar, desarrollar y evaluar en cada unidad (Extracto R. 29). 


\section{LA NUEVA ASIGNATURA DE EDUCACIÓN CIUDADANA EN CHILE}

Tabla 1. Ejemplos de opiniones de participantes sobre la pregunta 2.

\begin{tabular}{|c|c|}
\hline Opiniones positivas & Opiniones negativas \\
\hline $\begin{array}{l}\text { - El programa está bastante completo, tiene una estructura } \\
\text { que aborda los temas principales como el Estado, los } \\
\text { componentes, la Constitución y además los DD.HH. (R. 22) } \\
\text { - Es muy bueno, organizado, es coherente con las } \\
\text { necesidades e intereses de los estudiantes en la actualidad. } \\
\text { Los conceptos a trabajar son precisos y dan pie para } \\
\text { realizar actividades prácticas en el aula. }(R .48)\end{array}$ & $\begin{array}{l}\text { - Mediocre (R. 23) } \\
\text { - Es sesgado y reduccionista (R. 8) }\end{array}$ \\
\hline Opiniones positivas con aspectos negativos & Opiniones negativas con aspectos positivos \\
\hline $\begin{array}{c}\text { - Considero que la propuesta es interesante, pero que } \\
\text { necesita de mayor acompañamiento hacia el docente, } \\
\text { pues deja un espacio bastante amplio para abordar las } \\
\text { temáticas. Falta mayor precisión de los contenidos y } \\
\text { objetivos (R. 7) } \\
\text { - Tiene buenas intenciones, pero me parece muy impreciso, } \\
\text { vago y que al intentar enfocarse en habilidades pierde los } \\
\text { conceptos concretos y conceptuales, así como de aplicación } \\
\text { concreta (R. 30) }\end{array}$ & $\begin{array}{c}\text { A nivel general, por todo lo visto he notado que } \\
\text { el programa ministerial fue sacado de forma } \\
\text { apresurada., un poco sobre la marcha. Lo que } \\
\text { da alguna solución improvisada, esto como un } \\
\text { prejuicio. En cuanto a lo del programa me parece } \\
\text { bien orientado, en cuanto a pilares como "El Rol } \\
\text { del Estado, los DD.HH. y la función del Mercado" } \\
\text { Sobre la estructura, me parece que está bien } \\
\text { armado en cuanto (R. 16) }\end{array}$ \\
\hline
\end{tabular}

Respuestas neutrales

- No lo he revisado en la profundidad suficiente como para formarme una opinión (R.2)

- Aún muy general, para mi es difícil dar una respuesta sin el desarrollo práctico de la asignatura (R. 20)

Fuente: Elaboración propia.

La respuesta $\mathrm{N}^{\circ} 29$ corresponde a una visión positiva con aspectos negativos. Este tipo de respuesta habla de docentes que, a pesar de encontrar complejidades, como la falta de indicaciones ministeriales, son capaces de desarrollar respuestas de adaptación creativa (Ávalos, 2006), a partir de sus propias creencias para intentar cumplir con el programa de estudio.

Respecto a la segunda pregunta de investigación de este estudio ‘¿Qué tan capacitados se sienten los profesores y profesoras de Historia para impartir la asignatura de Educación Ciudadana considerando su formación inicial docente?', cabe recordar que las distintas investigaciones reportan insuficiencia de asignaturas orientadas a la enseñanza de Ciudadanía en Chile (Mardones et al. 2014; Agencia de Calidad de la Educación, 2016; Altamirano, 2018). Lo que correspondería a una insuficiencia de recursos académicos (Laura, 2005), aspecto que como vimos, incide en la implementación de nuevos currículos pudiendo transformarse en un aspecto de resistencia.

Al mirar las respuestas a la pregunta ‘¿Cree usted tener suficientes herramientas profesionales para hacer clases del curso de educación ciudadana?' vemos que las respuestas están mayoritariamente divididas entre quienes dicen tal vez (46\%) y quienes afirman tenerlas (44\%). El grupo que afirma no tenerlas es minoritario (10\%). Por otro lado, entre quienes afirman tener suficientes herramientas, resulta llamativo que varias respuestas señalan abiertamente no haber tenido formación inicial apropiada para la enseñanza de la asignatura, aspecto coincidente con las investigaciones al respecto:

En mi formación inicial la formación en Educación Ciudadana sólo fue un curso, situación insuficiente ante la necesidad de implementar un programa por dos años. 
No obstante, a través del tiempo y por formación profesional continua he cubierto esos vacíos conceptuales, también he tenido la posibilidad de crear programas propios en estas temáticas. En complemento, participé en la construcción del programa de formación ciudadana para el colegio. (R. 43)

Tal como se expresa en la respuesta $\mathrm{N}^{\circ} 43$, la formación continua es el aspecto que se menciona mayormente por parte de los y las docentes para superar la falta de conocimiento entre quienes aseguran tener suficientes herramientas para dictar el curso. Esto también coincide con lo señalado por Mardones y colaboradores (2014) referido a la disposición de los y las profesoras para perfeccionarse en función de la enseñanza de la ciudadanía. En ese sentido, vemos que la formación continua permite a los docentes superar la insuficiencia de formación inicial, que en este caso no se constituye en aspecto de resistencia.

Un aspecto interesante que emerge de las respuestas es que, no obstante, hay docentes que reportan la insuficiencia de formación inicial, también hay una minoría (23\%) que sostiene que sí tuvo una formación inicial apropiada para la enseñanza de la asignatura, lo que contrasta con las investigaciones antes expuestas. A este grupo se suma un docente que responde tal vez, afirmando haber recibido formación inicial pertinente. Al cruzar dichas respuestas de este grupo de profesores con el tiempo de ejercicio profesional vemos que casi todas, salvo un docente con 20 años de experiencia, corresponden a docentes que están entre los 1 y 3 años de ejercicio profesional. De este grupo sólo dos docentes están realmente dictando la asignatura. De ello podríamos inferir que la formación inicial que entregan las universidades efectivamente es escasa en cantidad de cursos, pero orientada a una comprensión más amplia y maximalista de la ciudadanía. No obstante, también es posible que el no estar dictando el curso les dé a estos profesionales una falsa percepción de suficiencia que solo se comprobará una vez que efectivamente tengan la necesidad de enseñar la asignatura.

Entre quienes responden tal vez y se encuentran actualmente dictando la asignatura, resulta interesante el hecho de que la principal justificación es que creen contar con los conocimientos, no obstante, de igual forma tienen incertidumbre. Una segunda justificación enunciada, corresponde a una insuficiente formación inicial, sin embargo, no quiere decir que no se sientan capacitados, sino inseguros al respecto. Luego con igual cantidad de menciones aparece la experiencia para dictar la asignatura.

Es posible inferir que los docentes, aun pudiendo tener las herramientas, tienen una percepción de que esta es insuficiente y de ahí la sensación de inseguridad.

Aunque gran parte de los y las docentes reconocen carencias en la formación universitaria, la experiencia y la formación continua aparecen como aspectos relevantes que permiten superar este déficit, sumado al hecho que consideran las herramientas para enseñar Historia, similares y por tanto extrapolables a esta disciplina, lo que los posiciona en una postura de adaptación creativa frente a la asignatura, más que en una lógica de resistencia.

Al mirar los resultados de la pregunta sobre la percepción referida a disponer suficientes recursos didácticos, emerge un aspecto interesante. Es igual la cantidad de docentes que plantean tenerlos versus los que no los tienen $(40 \%$ y un porcentaje minoritario (20\%) no tiene certeza al respecto. 
En función de los tipos de recursos que los y las docentes enuncian como pertinentes o necesarios para el desarrollo de la asignatura de Educación Ciudadana, se ha distinguido a partir de su descripción, dos grandes dimensiones. La primera de ellas, 'fuentes y soportes escritos y audiovisuales' (Tabla 2) que constituye la referencia a recursos de diversa índole que les permiten trabajar en la asignatura, sin connotar qué posibilidades y oportunidades de aprendizaje que entregan, donde existen ejemplos en torno a recursos como texto de estudio, recursos audiovisuales, o como vemos, asociados a soportes digitales y TICS.

Tabla 2. Ejemplos de recursos: fuentes y soportes escritos y audiovisuales.

- Algunos recursos pertinentes podría ser los vídeos, la revisión de los diarios virtuales. (R.7)

- Buenos textos escolares para todos. (R.5)

- Quizás sería bueno pensar en base de datos de archivos, películas, documentales, fotografías, notas de prensa, que permitan acceder a diversidad de recursos según los temas. (R.12)

Por otra parte, podemos visualizar aquellos recursos que entregan oportunidades para desarrollar habilidades y actitudes ciudadanas a partir de su trabajo -ya sea por medio de TICS o sin estas-, en las cuales podemos evidenciar un enfoque amplio y maximalista en términos de conceptualización y aprendizaje de la ciudadanía (Kerr, 1999, 2002). Lo anterior considera una comprensión profunda en términos conceptuales y desarrollo actitudinal respecto la perspectiva que entregan y a partir del desarrollo de habilidades como la participación, reflexión, pertenencia y valoración de la democracia. Tal como podemos visualizar en los ejemplos, pretende una comprensión de una ciudadanía activa, reflexiva y crítica, propio de una nueva comprensión curricular también presente en la asignatura (Ministerio de Educación, 2020).

De una parte, conocer una visión amplia y actualizada en torno a temáticas relativas a la ciudadanía activa y dialogante, entendiendo a los y las estudiantes y las comunidades educativas como agentes clave en la construcción de nuevas comunidades políticas. Por otra parte, se hace necesario manejar estrategias y dinámicas de aula que faciliten la participación activa de los y las estudiantes en la construcción del conocimiento. (Extracto R. 33)

Importante es destacar de estas referencias, la inclusión de estrategias que nos inviten a comprender una ciudadanía más amplia en esta nueva visión que constituye hoy el aprendizaje de la ciudadanía, siendo el aprendizaje basado en proyectos aquella que permitiría aprender en profundidad y vivenciar estas experiencias más democráticas de construcción del aprendizaje, foco también presente en la propuesta curricular de la asignatura:

Debates, utilizando fuentes históricas y sociales para su preparación. - Ensayos críticos. - Indagación digital (fuentes legales del congreso, prensa, etc.) - Proyectos de participación sobre desafíos del siglo XXI (por ejemplo, sobre el medio ambiente). (R. 3) 
Por último, destaca la mención a la interdisciplinariedad como estrategia de promoción de habilidades y competencias ciudadanas más amplias. La cual, no obstante, las tres acotadas referencias, permite visualizar una cantidad de docentes que entrega una potencialidad a los recursos en torno a la promoción y formación de competencias, comprensiones y actitudes ciudadanas más allá del trabajo en la asignatura.

Un enfoque transdisciplinario me parece fundamental, dada lo heterogéneo del estudiantado y las condiciones de profunda desigualdad que a todo nivel experimenta la sociedad chilena. (R. 20)

Cuando los y las docentes enuncian las principales dificultades para el desarrollo de la asignatura, destacan tres grandes obstaculizadores (Tabla 3). El primero de ellos, las estrategias de enseñanza y aprendizaje para la ciudadanía, por las cuales se comprende no solo las tensiones asociadas a una comprensión maximalista (en desmedro, de la minimalista)- donde la pregunta a la base es '¿cómo construir una ciudadanía crítica y reflexiva junto a estudiantes tal como plantea el currículo?'- sino también, cómo propiciar estas comprensiones en torno a la ciudadanía si no se ha formado parte de la elaboración de esta propuesta curricular. Tal como se visualiza en los ejemplos (Tabla 4) las dificultades que enuncian docentes surgen de las creencias en torno a la ciudadanía y expectativas de aprendizaje de esta, propiciando respuestas tanto de adaptación creativa, como de resistencia.

Tabla 3. Ejemplos de dificultades asociadas a estrategias de enseñanza y aprendizaje ciudadanía.

- Lograr estructurar un proyecto de participación ciudadana en el contexto local para hacer carne y práctica las enseñanzas, no es fácil (...). (R.4)

- Mi principal problema es a la hora de realizar preguntas de opinión fundamentadas a los estudiantes. (R.15)

- Hasta ahora, ha sido involucrar a todas las estudiantes en la discusión sobre algunos contenidos. (R. 12)

Fuente: Elaboración propia.

Tabla 4. Ejemplos de dificultades asociadas a la propuesta curricular de la asignatura.

- Los constantes cambios en el currículo y la poca precisión de los programas de estudio, esto implica que llevarlos al aula requiera de mucha inversión de tiempo en el análisis y la gestión de recursos e insumos. (R. 30)

- Creo que la novedad y el poco conocimiento del programa de la asignatura puede ser la principal dificultad.

Dominar el currículum es clave y eso requiere tiempo. (R.2)

- Contar con poco tiempo para su implementación. (R.50)

En relación con la tensión antes enunciada, encontramos una segunda dificultad planteada por el 20\% de docentes consultados, la cual corresponde a la visión que poseen de la propuesta curricular recién implementada. En este sentido, podemos ver cómo docentes visualizan esta como un problema, ya que al ser concebida como una propuesta emanada desde fuera (Fullan, 2002), no se considera como realmente facilitadora para promover el 
aprendizaje de una ciudadanía amplia en estudiantes, como tampoco idónea, en términos de los recursos que se posee para su desarrollo.

Como vemos, no solo los constantes cambios de los últimos años generan resistencia, sino, además, la cercana implementación desde su publicación. Elemento que se traduce finalmente, en una percepción resistente tanto por las posibilidades que entrega, como por el recurso tiempo del que se dispone para su comprensión e implementación (De Alba, 1991).

Asociado a estas condicionantes, surgen las tensiones (Tabla 5) en torno al contexto en que docentes se desempeñan, el cual se convierte en un aspecto de preocupación en tanto determina tensión y crítica respecto de las condiciones institucionales, las que a su vez, pueden constituir obstaculizadores.

Tabla 5. Ejemplos de tensiones en torno al contexto en torno al que se desempeñan

Limitación del colegio para realizar asambleas por ejemplo (limitación doctrinaria religiosa y política) (R39)

Considero que el gran desafío es el entablar acuerdos con equipos directivos que no necesariamente están culturalmente habituados a la participación de los y las estudiantes. (R 33)

La falta de trabajo colaborativo docente es una asignatura de historia, pero su enfoque demanda compromiso de todas las demás áreas. La formación ciudadana no se trabaja realmente de forma transversal. (R48)

Así es como podemos observar cuán determinante para docentes son las condiciones del contexto que posibilitan aprendizajes profundos en términos de ciudadanía, comprendiendo que se requiere de estas condiciones para posibilitar mejores oportunidades de aprendizaje para estudiantes.

Nuevamente surgen tensiones asociadas a la formación inicial que docentes indican requerida para implementar la asignatura, así es como existen referencias que apelan a la escasa formación inicial que poseen, que a pesar de no correlacionarse con su respuesta a cuán capacitados se sientes para impartir la asignatura, si se presenta como una dificultad de implementación en esta oportunidad. Dando cuenta así, de los insuficientes recursos académicos que poseen (Laura, 2005):

Creo que el principal es el desconocimiento y la poca capacitación a los profesores ya instalados en las aulas de clases. Una cosa es el dictar curso y la otra que realmente todos los profesores de Historia sean aptos para realizar el curso. (R16)

Por último, encontramos una dificultad referenciada por el 20\% de docentes, que no se relaciona con las tensiones comprendidas al instalar nuevos currículos, pero si, con las creencias y compresiones en torno a la ciudadanía y su aprendizaje. En este sentido, surge un nuevo actor, que estaba indirectamente referenciado en las respuestas anteriores, pero que al visualizar las dificultades -y como veremos, luego en las facilidades- es explícitamente indicado, el actor "estudiantes" (Tabla 6). 
Tabla 6. Ejemplos de dificultades asociadas a "estudiantes".

- Los estudiantes tienen otros intereses (video juegos, RRSS) (R. 44)

- (...) Por último, no es manifiesto el interés de los alumnos la temática del trabajo colectivo y el del preocuparse por estar informado de la temática política. (R. 24)

- En mi experiencia, una de las principales dificultades es la base conceptual previa de los estudiantes (...) (R. 41)

Es interesante que se explicite concretamente como una dificultad a estudiantes, cuando se ha visualizado en las opiniones anteriores, que se concibe -en general, por parte de docentes- una ciudadanía amplia, donde ciertamente los protagonistas son estos. Cabe preguntarnos entonces, ¿cuánto puede esta comprensión posibilitar la resistencia en la implementación de la nueva asignatura y su aprendizaje en profundidad?.

Cuando los y las docentes enuncian facilidades que poseen al realizar clases de Educación Ciudadana, destaca ampliamente la percepción que poseen de la asignatura y las potencialidades que visualizan en torno al aprendizaje de una ciudadanía activa y crítica junto a sus estudiantes. En esta pregunta, más del 70\% de las menciones da cuenta de posibilidades y oportunidades entregadas por el aprendizaje de la ciudadanía, así como la motivación que genera en estudiantes su desarrollo a partir del trabajo con sus contenidos, habilidades y actitudes (Tabla 7).

Tabla 7. Ejemplos de percepciones en torno a potencialidades.

(...) es tener claro que la educación ciudadana no se aprende porque se lee la constitución o se señalan las características de la democracia, sino porque se viven experiencias de ejercicio ciudadano, todo el rato vinculando la experiencia de la comunidad escolar como comunidad democrática. (R. 12)

- (...) He notado más interés en muchos chicos sobre sus derechos ciudadanos. Quieren participar y ser actores dentro de la ciudadanía, sobre todo en lugares como Nogales que presenta problemas graves por la falta y robo de agua. (Extracto R.16)

Como podemos visualizar, no solo se evidencia una comprensión amplia y maximalista de la ciudadanía (Kerr, 1999, 2002), sino además comprensiva del nuevo foco enunciado por la propuesta curricular de la asignatura (Ministerio de Educación, 2020), al evidenciar una educación ciudadana como forma de vida, capaz de desarrollar habilidades y actitudes, más que, contenidos conceptuales memorísticos. Lo que, a su vez, evidencia una traducción del currículo a partir de propias creencias de docentes (Wallace \& Priestley, 2011) al centralizar este proceso de enseñanza y aprendizaje en estudiantes, volviéndolos también, sujetos activos tanto de su aprendizaje como de su ciudadanía, conforme no se aprende automáticamente a serlo y es en la escuela donde se accede a un 'nosotros' (Cox y Castillo, 2015).

En cuanto a los recursos, podemos visualizar ejemplos que recorren desde elementos o condiciones de acceso materiales, como aulas espaciosas, conectividad, textos de estudios, a condiciones institucionales (Tabla 8), que a pesar de que previamente se visualizan como 


\section{LA NUEVA ASIGNATURA DE EDUCACIÓN CIUDADANA EN CHILE}

Tabla 8. Ejemplos de facilidades asociadas a "recursos".

- El libro de texto entregado por el ministerio de educación cuenta con muchos recursos que se pueden utilizar en clases. (R. 9)

- Además de aulas espaciosas y buen clima de aula para generar constante debate y opinión (R. 47)

Tabla 9. Ejemplos de facilitadores relacionads con la formación inicial

Mi formación disciplinaria (historiografía) de pregrado que me permite investigar y profundizar en temas nuevos y actualizar mis conocimientos en los distintos campos del desempeño pedagógico. (R. 20)

(...) En relación a esto, es pertinente destacar que la formación en conocimientos didácticos y pedagógicos de la universidad nos aporta una enorme gama de herramientas para llevar a cabo esta labor como docentes. (Extracto R. 21)

obstaculizadores cuando se piensa el aprendizaje de la ciudadanía. También logra distinguirse como una facilidad para instalar una propuesta que promueva mayores oportunidades de aprendizaje de esta. Así es como estas posibilidades tanto como condiciones o recursos materiales para la enseñanza, nos invitan a pensar en que existan respuestas de adaptación creativa (Ávalos, 2006) al implementar la asignatura por parte de docentes.

Aún cuando la investigación sobre el tema reporta deficiencias en la formación inicial docente sobre la temática (Mardones, et al. 2014; Agencia de la Calidad de la Educación, 2016; Altamirano, 2018), vemos como un grupo minoritario lo releva como un facilitador en la implementación de la asignatura de Educación Ciudadana (Tabla 9). Cabe destacar que este grupo corresponde en su mayoría a profesores que se insertaron al sistema escolar en promedio dos años atrás.

A partir de estos ejemplos, es importante preguntarnos cuánto de esta formación inicial y conocimientos profesionales posibilitará concretamente una adaptación creativa o cómo se traducirá efectivamente en un aprendizaje de una ciudadanía amplia junto a estudiantes.

\section{CONCLUSIONES}

A pesar de que la asignatura de Educación Ciudadana había sido anunciada con anticipación, lo cierto es que el plan de estudio definitivo no se publicó hasta los primeros días de marzo de 2020, justamente al momento en que debía comenzar a implementarse, aspecto que emerge como obstaculizador en el discurso de varios de los y las docentes encuestados. A ello, se suma el hecho de que la formación inicial de profesores en Chile no ofrece suficientes cursos orientados a la enseñanza de la ciudadanía, provocando incertidumbre y tensión al implementarle.

$\mathrm{Al}$ mirar las preguntas de investigación vemos que los y las docentes encuestados tienen una visión favorable de la asignatura, fundamentalmente porque la esperaban hace tiempo y debido a que entrega oportunidades de aprendizaje ampliamente valoradas por docentes y estudiantes, en términos de una ciudadanía tendiente a la formación de habilidades y actitudes.

Respecto a la segunda pregunta de investigación vemos que la mayoría de los docentes tienen dudas acerca de tener suficientes herramientas profesionales para la enseñanza 
de la asignatura, aunque un porcentaje importante afirma tenerlas. Aun cuando desde su percepción les faltan herramientas, y en algunos casos, conocimiento disciplinar producto de una falencia en su formación inicial docente, lo cierto es que la formación continua, sumada a la experiencia, les da a los y las docentes la posibilidad de dictar la asignatura desde una posición de adaptación creativa, utilizando para ello las herramientas de que disponen.

Por último, cuando los y las docentes indican cuáles son sus dificultades y facilidades para la implementación de la asignatura destacan en relación a las primeras, las tensiones asociadas a recursos académicos (como la formación inicial); condiciones de implementación de la propuesta (como el acotado tiempo que poseen); condiciones institucionales (como el trabajo colaborativo); y principalmente, inconvenientes asociados a la comprensión, en términos de aprendizaje de la ciudadanía que buscan promover, puesto que en su mayoría, los y las docentes conciben una asignatura que posibilita oportunidades de aprendizaje de una ciudadanía crítica, reflexiva y como forma de vida en la escuela, pero paras las cuales carecen de herramientas o por lo menos, presentan dificultades. En contraposición, y aunque pareciera contradictorio, los mismos factores son enunciados como facilitadores, a saber, recursos, formación inicial y conocimientos docentes, y, sobre todo, la comprensión de una ciudadanía activa. Aun cuando los y las docentes visualizan tensiones, explicitan una adaptación creativa a esta implementación profundamente imbricada a la oportunidad de aprendizaje que le entregan a esta comprensión amplia y maximalista de la ciudadanía en la escuela.

\section{REFERENCIAS}

Agencia de Calidad de la Educación (2016). Formación ciudadana en el sistema escolar chileno: una mirada a las prácticas actuales y recomendaciones de mejora. Santiago: ACE. Recuperado de: http://archivos.agenciaeducacion.cl/liderazgo-motivacion-lectora/ FORMACION_CIUDADANA.pdf

Altamirano, M. (2018). Pensamiento y prácticas del profesorado de Historia, Geografía y Ciencias Sociales sobre la formación ciudadana en Chile. Tesis de Doctorado, Universidad Autónoma de Barcelona, Barcelona, España. Recuperado de:https://www. tdx.cat/handle/10803/665822\#page=1

Ávalos, B. (2006). Currículo y desarrollo profesional docente. Revista PRELAC, 3, 104-111

Bantwini, B. (2010). How teachers perceive the new curriculum reform: Lessons from a school district in the Eastern Cape Province, South Africa. International Journal of Educational Development, 30, 83-90.

Bascopé, M., Cox, C., \& Lira, R. (2015). Tipos de ciudadano en los currículos del autoritarismo y la democracia. En: Cox, C., \& Castillo, J. (2015). Aprendizaje de la ciudadanía. Contextos, experiencias y resultados. Santiago: Ediciones Universidad Católica de Chile.

Calderhead, J. (1996). Teachers: Beliefs and knowledge. En D. Berliner \& R. Calfee (Eds.), Handbook of educational psychology (pp. 709-725). New York: Macmillan.

Casas, J., Repullo, J., \& Donado, J. (2003). La encuesta como técnica de investigación. Elaboración de cuestionarios y tratamiento estadístico de los datos (I). Atención Primaria, 31(8), 
527-538. http://doi.org/10.1016/S0212-6567(03)70728-8

Consejo Asesor Presidencial contra los Conflictos de Interés, el Tráfico de Influencias y la Corrupción (2015). Informe Final. Recuperado de: http://consejoanticorrupcion.cl/ informe/

Cox, C. (2011). Currículo Escolar de Chile: génesis, implementación y desarrollo. Revue International de Education de Sevres, 56, 1-9.

Cox, C. \& García, C. (2015) Objetivos y contenidos de la Formación Ciudadana escolar en Chile 1996-2013: tres currículos comparados. En: Cox, C., \& Castillo, J. (2015). Aprendizaje de la ciudadanía. Contextos, experiencias y resultados. Santiago: Ediciones Universidad Católica de Chile.

De Alba, A. (1991) Currículum: Crisis, mito y perspectiva. Centro de estudios sobre la Universidad. Buenos Aires: Miño y Dávila Editores.

Edwards, R. (2010). Translating the Prescribed into the Enacted Curriculum in College and School. En T. Fenwick \& R. Edwards (Eds.), Actor-network theory in education (pp. 2339). New York: Routledge.

Fives, H., \& Buehl, M. (2016). Teachers' Beliefs, in the Context of Policy Reform. Policy Insights from the Behavioral and Brain Sciences, 3(1), 114-121. http://doi. org/10.1177/2372732215623554

Fullan, M. (2001). Leading in a culture of change. San Francisco, CA: Jossey-Bass.

Fullan, M. (2002). El significado del cambio educativo: un cuarto de siglo de aprendizaje. Profesorado, revista de currículum y formación del profesorado, 6(1-2), 1-14. Recuperado de: http://www.ugr.es/ recfpro/rev61ART1.pdf

Handal, B., \& Herrington, A. (2003). Mathematics teachers` beliefs and curriculum reform. Mathematics Educational Research Journal, 15(1), 59-69.

Hernández, R., Fernández, C., \& Baptista, P. (2014). Metodología de la investigación. (6ª. Ed). México D.F.: McGraw-Hill.

Kerr, D. (1999). Citizenship education in the curriculum: An international review. The School Field, 10(3-4), 5-32.

Kerr, D. (2002) Comparative and international perspectives on citizenship education. En J. Arthur, \& H. Cremin (Eds.), Debates in Citizenship Education (pp. 17-31). Routledge: Londres.

Kerr, D. (2015) Ciudadanía a nivel nacional, regional e internacional: una revisión de enfoques, investigaciones y debates. En: Cox, C., \& Castillo, J. (2015). Aprendizaje de la ciudadanía. Contextos, experiencias y resultados. Santiago: Ediciones Universidad Católica de Chile.

Laura, L. (2005). Curriculum. Reflexiones sobre los obstáculos para su formulación e implementación. Revista Argentina de Sociología, 3(5), 246-265. Recuperado de: https:// www.redalyc.org/articulo.oa?id=269/26930513

Ley 20.911. Crea el plan de formación Ciudadana para los establecimientos educacionales reconocidos por el Estado. 2 de abril de 2016. Ministerio de Educación, Chile. Recuperado de: https://www.bcn.cl/leychile/navegar?idNorma=1088963

Mardones, R. Cox, C. Farías, A. García, C. (2014) Currículos comparados, percepciones docentes y formación de profesores para la formación ciudadana: tendencias y proposiciones de 
mejoramiento. Concurso Políticas Públicas, 215-246.

Ministerio de Edycación (2019) Bases Curriculares $3^{\circ}$ - $4^{\circ}$ Medio. Santiago: Unidad de Currículum y Evaluación.

Ministerio de Educación (2020) Programa de estudio educación ciudadana 3 Medio. Santiago: Unidad de Curriculum y Evaluación.

Pajares, M. (1992). Teachers' beliefs and educational research: Cleaning up a messy construct. Review of educational research, 62(3), 307-332.

Rocco, L., \& Oliari, N. (2007). La encuesta mediante internet como alternativa metodológica. VII Jornadas de Sociología. Buenos Aires: Facultad de Ciencias Sociales, Universidad de Buenos Aires.

Rodríguez, J., \& Solis, C. (2017). Creencias docentes: Lo que se hace en el aula es consecuencia de lo que se piensa. Propósitos y Representaciones, 5(1) 7-20. http://dor.org/10.20511/ pyr2017.v5n1.155

Roehrig, G., Kruse, R., \& Kern, A. (2007). Teacher and school characteristics and their influence on curriculum implementation. Journal of research on in scienceteaching, 44(7) 883907.

Sánchez, M., Delgado, M., \& Santos, M. (2013). El proceso de la investigación cualitativa. Manual de procedimiento: ejemplificación con una tesis doctoral.Valladolid: Edintras.

Shulman, L. (1986). Those who understand: Knowledge growth in teaching. Educational Researcher, 15(2), 4-14.

Spillane, J., Reiser, B., \& Reimer, T. (2002). Policy Implementation and Cognition: Reframing and Refocusing Implementation Research. Review of EducationalResearch, 72(3), 387431. Recuperado de: www.jstor.org/stable/3515992

Tham, M. (2014). La implementación del currículum escolar de Formación Ciudadana. Tesis para optar al grado de Magíster en Ciencias Sociales con mención en Sociología de la Modernización, Universidad de Chile, Santiago, Chile. Recuperado de http://repositorio. uchile.cl/handle/2250/140283

Thomas, G. (2017). How to do your research project. A guide for students. London: Sage.

Wallace, C., \& Priestley, M. (2011). Teachers beliefs and the mediation of curriculum innovation in Scotland: A socio-cultural perspective on professional development and change. Journal of Curriculum Studies, 43(3) 357-381. 